\title{
Dosimetric Comparison of Anisotropic Analytical Algorithm and Acuros XB in Stereotactic Body Radiotherapy and Effect of Calculation Grid Size
}

\author{
Aydın ÇAKIR \\ Department of Radiation Oncology, Memorial Şişli Hospital, İstanbul-Turkey
}

\begin{abstract}
OBJECTIVE
This study aimed to determine the dose differences between Acuros XB (AXB) and anisotropic analytical algorithm (AAA) for patients with liver cancer who underwent stereotactic body radiotherapy (SBRT) and to investigate the dose-related effect of dose calculation grid size (CGS).

\section{METHODS}

SBRT treatment was planned for 10 patients with liver cancer using 1-mm and 2.5-mm CGS with AAA and AXB algorithms with $10 \mathrm{MV}$ flattening filter-free (FFF) rays in Varian Trubeam STx.

\section{RESULTS}

When AAA 1-mm CGS plans and AAA 2.5-mm CGS plans were compared, $4 \%$ difference was observed; when AXB 1-mm CGS plans and AXB 2.5-mm CGS plans were compared, 1\% difference was found. No significant difference was found between plans with AAA 1-mm CGS and plans with AXB 1-mm CGS ( $\mathrm{p}>0.05)$. On the other hand, there was a significant difference between plans with AAA 2.5mm CGS and plans with AXB 2.5-mm CGS $(\mathrm{p}<0.05)$.
\end{abstract}

\section{CONCLUSION}

As a result of the study, it was seen that the AXB algorithm gave more stable results than the AAA one in different intensity body regions. High doses are administered in a small number of fractions in SBRT. For SBRT, 1-mm CGS should be selected for calculation accuracy.

Keywords: Anisotropic analytical algorithm; acuros XB; calculation grid size.

Copyright $\odot$ 2017, Turkish Society for Radiation Oncology

\section{Introduction}

In advanced radiotherapy methods such as intensity modulated radiotherapy (IMRT) and volumetric modulated arc radiotherapy (VMAT), sharp dose drops can be achieved after the tumor volume; desired high doses are given to target volumes determined using different imaging techniques. In stereotactic radiosurgery (SRS) and stereotactic body radiotherapy (SBRT), it is very important to give prescribed doses to target volumes with geometric uncertainty under the millimeter. In SRS/SBRT, high doses are administered in a small number of fractions. In these treatments, accuracy can be achieved using precise calculation of the algorithm used.

In the treatment planning system of Eclipse 13.0 (Varian Medical Systems, Palo Alto, CA), the anisotro- 
pic analytical algorithm (AAA) is commonly used for dose calculation.

Recently, in the dosimetric study performed by many investigators, it has been reported that AAA calculated the calculated dose significantly inaccurate.[112] Particularly, it has been observed that in the transition from tissue to air, dose is incorrectly calculated near two mediums. Varian (Varian Medical Systems, Palo Alto, CA) has introduced a new dose calculation algorithm called Acuros XB (AXB), a computational algorithm for clinical use. AXB uses a sophisticated technique to solve the Linear Boltzmann Transport Equation (LBTE), and it provides the correct approach for calculating patient dose from heterogeneous sources entirely of lung, bone, air, and different density implants. LBTE describes the macroscopic behavior of the radiation beam in its environment.[2-4]

There are many studies in the literature that dosimetrically compared the AAA and AXB algorithms.[3-8] The dose difference between the two algorithms results from parameters such as energy of the incoming beam, field size, and electron density of the medium.

However, studies have reported that the calculation grid size (CGS) is associated with dose changes. The difference between AAA and AXB due to different uses is not known to affect SBRT treatments, and this effect requires further investigation.

The dosimetric effect of AXB in the SBRT plan for lung cancer has little information on this. With advancing technological facilities, manufacturers of linear accelerator devices offer both flattened (FF) and unflattened (FFF) beams together. SBRT treatments can be applied to patients in a shorter time because of increased dose rate due to FFF beams.

This study aimed to investigate the calculated differences between AXB and AAA and the dose-related effect of dose CGS on SBRT treatments for planned liver cancer with FFF beams.

\section{Materials and Methods}

\section{Eclipse Treatment Planning System}

Eclipse TPS 13.0 (Varian, Palo Alto, California, USA) is designed to make 3D CRT, IMRT, VMAT, SRS/SBRT, and electron schemes. In the Eclipse treatment planning system in our clinic, pencil beam convolution and analytical anisotropic algorithm (AAA) are performed with dose volume optimization, plan geometry optimization, progressive resolution optimization, multiresolution dose optimization (MRDC), and Acuros XB (AXB) dose calculation algorithms.

\section{Analytical Anisotropic Algorithm}

The AAA dose calculation model is a $3 \mathrm{D}$ pencil beam and convolution superposition algorithm consisting of separate models for electrons emitted from primary photons, scattered photons, and beam modulators (primary collimator, beam straightening filter, and wedge filter).[10] The functional forms that form the basic physical quantities initiate a process by adding device properties to the account. This often leads to a noticeable reduction in the computation required for such algorithms. Tissue heterogeneities are anisotropically accounted for using photon scattering kernels in multiple lateral directions in a 3D neighborhood. The final dose distribution is formed by superimposing the photon- and electron-initiated process.

\section{Acuros XB Algorithm}

The AXB algorithm was developed for two strategic needs such as accuracy and speed in external photon beam treatment planning. AXB uses a sophisticated technique to solve the LBTE and fully exploits heterogeneity in patient mortality from lung, bone, air, and non-biological implants.

Instead of Boltzman Transport Equation, which describes the macroscopic behavior of radiation particles, LBTE, its linear form, assumes that the particles in the environment interact with each other and the external magnetic field. [2-4] There are two solution approaches that try to explain LBTE. One of them is the Monte Carlo method, which does not clearly solve the commonly known LBTE and produces indirect solutions for LBTE. Second one is solving LBTE using numerical methods.

Monte Carlo and LBTE solution methods produce close results but fail to produce clear solutions.[2-4] The mistakes of Monte Carlo System are random and result from the fact that a limited number of particles are simulated. Systematic faults can occur when using Monte Carlo methodologies to expedite the solution time.

The source model of the AXB algorithm used in the Eclipse TPS uses the existing AAA source model. In this model; primary photons, out-of-focus photons, contaminant electrons, and photons scattered from the wedge.

The AXB algorithm uses knowledge of the mass concentration obtained in the CT images of each voxel for dose calculation. The calculation difference between the AAA and AXB algorithms depends on the beam energy, field size, and material density. 


\begin{tabular}{|c|c|c|c|c|c|c|}
\hline \multirow[t]{2}{*}{ Table 1} & \multirow[t]{2}{*}{ s } & \multirow{2}{*}{\multicolumn{2}{|c|}{$\begin{array}{l}\text { Id critical organs calculated fo } \\
\qquad \begin{array}{l}\text { AAA } 2.5 \\
\text { mm }\end{array}\end{array}$}} & \multirow[b]{2}{*}{$\begin{array}{l}\text { AXB } \\
2.5 \mathrm{~mm}\end{array}$} & \multirow[b]{2}{*}{$\begin{array}{l}\text { AAA } \\
1 \mathrm{~mm}\end{array}$} & \multirow[b]{2}{*}{ AXB 1mm } \\
\hline & & & & & & \\
\hline \multirow[t]{3}{*}{ PTV } & Dmin & cGy & 5541 & 5489 & 5738 & 5458 \\
\hline & Dmax & cGy & 6323 & 6381 & 6363 & 6388 \\
\hline & Dmean & cGy & 6098 & 6090 & 6098 & 6100 \\
\hline Spinal cord & Dmax & cGy & 641 & 720 & 629 & 1005 \\
\hline \multirow[t]{2}{*}{ Heart } & Dmax & cGy & 696 & 770 & 868 & 734 \\
\hline & Dmean & cGy & 111 & 107 & 115 & 110 \\
\hline \multirow[t]{4}{*}{ Lung } & V5 & $\%$ & 7.8 & 8.3 & 7.7 & 8.2 \\
\hline & V10 & $\%$ & 2.8 & 3.4 & 2.6 & 2.5 \\
\hline & V20 & $\%$ & 0.2 & 1.1 & 0.1 & 0.1 \\
\hline & Dmean & cGy & 114 & 181 & 170 & 170 \\
\hline Bilateral Kidney & Dmean & cGy & 97 & 87 & 93 & 86 \\
\hline Small Intestine & Dmax & cGy & 2078 & 2210 & 2255 & 2276 \\
\hline
\end{tabular}

\section{Varian TrueBeam STx Linear Accelerators}

Varian TrueBeam STx is a radiotherapy device using 3D Conformal, IMRT, IGRT, VMAT, stereotactic radiosurgery (SRS), and stereotactic body radiotherapy (SBRT). This linear accelerator is designed as a digital linear accelerator with $6 \mathrm{MV}, 10 \mathrm{MV}, 15 \mathrm{MV}$ flattening filters (FF) and $6 \mathrm{MV}$ and $10 \mathrm{MV}$ flattening filter-free (FFF) beams. The dose range of filtered beams is 100 $600 \mathrm{MU} / \mathrm{min}, 400-1400 \mathrm{MU} / \mathrm{min}$ for unfiltered FFF beams is $6 \mathrm{MV}$, and $400-2400 \mathrm{MU} / \mathrm{min}$ for $10 \mathrm{MV}$ FFF. The maximum area dimensions used for active MLC with minimum $0.5 \times 0.5 \mathrm{~cm}$ and maximum $40 \times 40 \mathrm{~cm}$ area dimensions are $22 \times 40 \mathrm{~cm}$.

TrueBeam STx linear accelerator has a multileaf (MLC) consisting of 120 tungsten materials. MLCs are $2.5 \mathrm{~mm}$ thick in the isocenter and have 32 pairs of MLC and 28 pairs of 5-mm MLC that surround them from the outside. The tongue-and-groove effect has the same design as the Millennium MLC and the High Definition MLC (HD-MLC) in terms of rounded leaf edge properties. The radius of curvature of the Millennium $120 \mathrm{MLC}$ is $8 \mathrm{~cm}$, while the radius of curvature of HDMLC is $16 \mathrm{~cm}$. HD-MLC, irregularly shaped areas of 40 $\mathrm{cm}$ wide and $22 \mathrm{~cm}$ long can be formed.

We recruited 10 patients with liver cancer who were referred to our clinic and their CT data was used. Treatment plans were generated using the volumetric modulated arc (VMAT) SBRT planning method have been used two partial Arc angles with 10 MV FFF beams of Varian TrueBeam STx. For each patient, four treatment planning were done using AAA and AXB algorithms using 1 and $2.5 \mathrm{~mm}$ calculation grid (CGS), respectively.

\section{Results}

The dosimetric results of our plans for SBRT planning for liver disease are shown in (Table 1). In Table 1, doses of PTV minimum, maximal, and Dmean, spinal cord maximal dose, ipsilateral lung V5, V10, V20, and Dmean doses, bilateral kidney Dmean and small intestine Dmax doses were calculated for two AAA and AXB algorithms.

Figures la-d show the mean dose volume histograms for PTV and critical organs compared with those for 1 and $2.5 \mathrm{~mm}$ CGS plans. The dose distribution of SBRT treatment planning samples calculated using 1 $\mathrm{mm}$ and $2.5 \mathrm{~mm}$ CGS for both algorithms (AAA and $\mathrm{AXB}$ ) is shown in Figures 2a-d.

In the case of spinal cord, the AXB algorithm predicted a higher dose than the AAA algorithm. The dose change on the spinal cord was statistically significant $(p<0.05)$. However, the ipsilateral lung V5 and V10 are statistically higher in the AAA algorithm than in the AXB algorithm. 


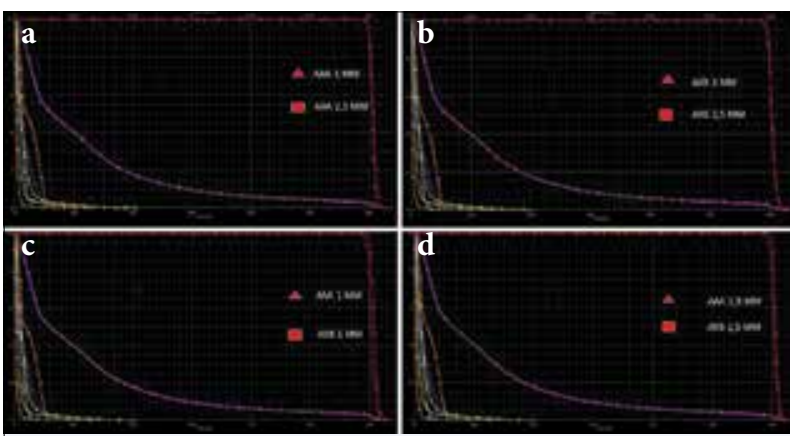

Fig. 1. (a) Dose volume histograms with AAA $1 \mathrm{~mm}$ and AAA $2.5 \mathrm{~mm}$ CGS. (b) Dose volume histograms with AXB $1 \mathrm{~mm}$ and AXB $2.5 \mathrm{~mm}$ CGS. (c) Dose volume histograms with AAA 1-mm CGS and AXB 1-mm CGS. (d) Dose volume histograms with AAA 2.5-mm CGS and AXB 2.5-mm CGS

There was a significant difference between PTV minimum doses of $1 \mathrm{~mm}$ CGS and $2.5 \mathrm{~mm}$ CGS AAA $(\mathrm{p}<0.05)$, whereas AXB plan with $1 \mathrm{~mm}$ CGS and AXB plans with $2.5 \mathrm{~mm}$ CGS showed close results ( $>0.05$ ). Considering the Dmax and Dmean doses for the heart, AAA and AXB with 1 and $2.5 \mathrm{~mm}$ CGS were not significantly associated with all plans $(\mathrm{p}>0.05)$. This is due to the fact that the AXB algorithm does not provide enough information about out-of-field side doses.

In the case of Dmean doses of ipsilateral lung doses, there was a significant difference between AAA plans with $1 \mathrm{~mm}$ CGS and $2.5 \mathrm{~mm}$ CGS $(\mathrm{p}<0.05)$. Likewise, differences between AXB plans with $2.5 \mathrm{~mm}$ CGS were significant $(\mathrm{p}<0.05)$. No significant results were found between AAA with $1 \mathrm{~mm}$ CGS and AXB plans with 1 mm CGS ( $\mathrm{p}>0.05)$.

There was a significant difference between bilateral renal doses of 1-mm CGS AAA and AXB plans and 2.5-mm CGS AAA and AXB $(\mathrm{p}<0.05)$.

When we examined the small intestinal Dmax doses in our study, no significant difference was found between 1-mm CGS plans and 2.5-mm CGS plans ( $\mathrm{p}>0.05)$.

The most interesting aspect of the work is that there is a $1 \%$ difference between AAA plans with 2.5-mm CGS and AXB plans with 2.5-mm CGS, and 5\% difference between AAA plans with 1-mm CGS and AXB plans with 1-mm CGS.

\section{Discussion}

In stage III patients with $\mathrm{HCC}$, using the 6-MV rays, $\mathrm{AXB}$ and $\mathrm{AAA}$ were compared with each other to ob-


Fig. 2. (a) AAA isodose distribution for $1 \mathrm{~mm}$. (b) AAA isodose distribution for $2.5 \mathrm{~mm}$. (c) Isodose distribution for AXB $1 \mathrm{~mm}$. (d) Isodose distribution for AXB $2.5 \mathrm{~mm}$. 
tain a slightly higher mean dose with the AXB algorithm in lung tissue.[13] Fogliata et al. in their study supports our study, in which our computed ipsilateral lung dose was calculated to be $114 \mathrm{cGy}$ with $2.5 \mathrm{~mm}$ AAA versus $181 \mathrm{cGy}$ with $2.5 \mathrm{~mm}$ AXB. In our study, there is a significant difference between $\mathrm{AAA}$ and $\mathrm{AXB}$ plans for 2.5-mm CGS ( $\mathrm{p}<0.05)$.

In their study, Kan et al.[14] found that AXB had a $1 \%$ higher dose than AAA for air trapping-included nasopharyngeal carcinoma treatment plans using IMRT and RapidArc techniques.

The difference between AAA and AXB is interesting as CGS has also contributed to the correct dose calculation. CGS is associated with the estimate and calculation accuracy. Kan et al.[14] showed a significant improvement in dose accuracy of AXB with 1-mm CGS. The smaller grid resolution reduces the average effect and results in a better sampling of the structure voxels. They showed that the dose difference in PTV was greater between the two algorithms in $2.5-\mathrm{mm}$ CGS for $6 \times \mathrm{FFF}$ and $10 \times \mathrm{FFF}$ beams.

In addition, Kan et al.[14] suggests that 1-mm CGS should be chosen for stereotactic plans, especially for low density tissue regions contained by PTV instead of 2.5-mm CGS.

Chung et al.[15] and Mittauer et al.[16] showed that CGS was effective on dose estimation for head and neck treatments. Ong et al.[17] demonstrated that 1-mm CGS accounts for a more accurate dose compared to AAA calculations with 2.5-mm CGS.

In our study, we found that there were $4 \%$ difference between AAA 1-mm CGS plans and AAA 2.5-mm CGS plans, whereas AXB 1-mm CGS plans and AXB 2.5-mm CGS plans had 1\% difference.

Regarding the PTV minimum doses, it was seen that there was a $5 \%$ difference between AAA plans with 1-mm CGS and AXB plans with 1-mm CGS. This may be the reason for the preference of the AXB algorithm to reduce the PTV dose during treatment planning. The effect of the dose difference between the two algorithms will be another area of interest for us. Our other work will focus on the difference between the two algorithms for different energy stages, focusing on the lung SBRT where small areas and air spaces are located.

In conclusion, SBRT treatments administer high doses in a small number of fractions. The accuracy of calculation related the accuracy of these treatments; it is necessary to ensure the dose response in the critical regions of the algorithm used.[18] The presence of different concentrations of tissue in the human body does not have a linear curve on the dose-response relation- ship. This effect should be investigated in detail before each clinical use.

\section{Disclosure Statement}

The authors declare no conflicts of interest.

Ethics Committee Approval: This study was conducted inaccordance with local ethical rules.

Peer-review: Externally peer-reviewed.

Conflict of Interest: None declared.

\section{References}

1. Fraass BA, Smathers J, Deye J. Summary and recommendations of a National Cancer Institute workshop on issues limiting the clinical use of Monte Carlo dose calculation algorithms for megavoltage external beam radiation therapy. Med Phys 2003;30(12):3206-16.

2. Vassiliev ON, Wareing TA, McGhee J, Failla G, Salehpour MR, Mourtada F. Validation of a new gridbased Boltzmann equation solver for dose calculation in radiotherapy with photon beams. Phys Med Biol 2010;55(3):581-98.

3. Bush K, Gagne IM, Zavgorodni S, Ansbacher W, Beckham W. Dosimetric validation of Acuros XB with Monte Carlo methods for photon dose calculations. Med Phys 2011;38(4):2208-21.

4. Hoffmann L, Jørgensen MB, Muren LP, Petersen JB. Clinical validation of the Acuros XB photon dose calculation algorithm, a grid-based Boltzmann equation solver. Acta Oncol 2012;51(3):376-85.

5. Tillikainen L, Helminen H, Torsti T, Siljamäki S, Alakuijala J, Pyyry J, et al. A 3D pencil-beam-based superposition algorithm for photon dose calculation in heterogeneous media. Phys Med Biol 2008;53(14):3821-39.

6. Tillikainen L, Siljamäki S, Helminen H, Alakuijala J, Pyyry J. Determination of parameters for a multiplesource model of megavoltage photon beams using optimization methods. Phys Med Biol 2007;52(5):144167.

7. Han T, Mikell JK, Salehpour M, Mourtada F. Dosimetric comparison of Acuros XB deterministic radiation transport method with Monte Carlo and model-based convolution methods in heterogeneous media. Med Phys 2011;38(5):2651-64.

8. Fogliata A, Nicolini G, Clivio A, Vanetti E, Cozzi L. Dosimetric evaluation of Acuros XB Advanced Dose Calculation algorithm in heterogeneous media. Radiat Oncol 2011;6(1):82. 
9. Fogliata A, Nicolini G, Clivio A, Vanetti E, Cozzi L. On the dosimetric impact of inhomogeneity management in the Acuros XB algorithm for breast treatment. Radiat Oncol 2011;6:103.

10. Robinson D. Inhomogeneity correction and the analytic anisotropic algorithm. J Appl Clin Med Phys 2008;9(2):2786.

11. Breitman K, Rathee S, Newcomb C, Murray B, Robinson D, Field C, et al. Experimental validation of the Eclipse AAA algorithm. J Appl Clin Med Phys 2007;8(2):76-92.

12. Van Esch A, Tillikainen L, Pyykkonen J, Tenhunen M, Helminen $\mathrm{H}$, Siljamäki $\mathrm{S}$, et al. Testing of the analytical anisotropic algorithm for photon dose calculation. Med Phys 2006;33(11):4130-48.

13. Fogliata A, Nicolini G, Clivio A, Vanetti E, Cozzi L. Critical appraisal of Acuros XB and Anisotropic Analytic Algorithm dose calculation in advanced nonsmall-cell lung cancer treatments. Int J Radiat Oncol Biol Phys 2012;83(5):1587-95.
14. Kan MW, Leung LH, Yu PK. Verification and dosimetric impact of Acuros XB algorithm on intensity modulated stereotactic radiotherapy for locally persistent nasopharyngeal carcinoma. Med Phys 2012;39(8):4705-14.

15. Chung H, Jin H, Palta J, Suh TS, Kim S. Dose variations with varying calculation grid size in head and neck IMRT. Phys Med Biol 2006;51(19):4841-56.

16. Mittauer K, Lu B, Yan G, Kahler D, Gopal A, Amdur R, et al. A study of IMRT planning parameters on planning efficiency, delivery efficiency, and plan quality. Med Phys 2013;40(6):061704.

17. Ong CL, Cuijpers JP, Senan S, Slotman BJ, Verbakel WF. Impact of the calculation resolution of AAA for small fields and RapidArc treatment plans. Med Phys 2011;38(8):4471-9.

18. Huang B, Wu L, Lin P, Chen C. Dose calculation of Acuros XB and Anisotropic Analytical Algorithm in lung stereotactic body radiotherapy treatment with flattening filter free beams and the potential role of calculation grid size. Radiat Oncol 2015;10:53. 